\title{
Decisions regarding use of urodynamic studies in stress urinary incontinence must be made on a case-by-case basis
}

\author{
Jerzy B. Gajewski, MD \\ Department of Urology, Dahhousie University, Halifax, NS, Canada
}

Cite as: Can Urol Assoc J 2017;11 (6Supp12):S1 16. hitp://dx.doi.org/10.5489/cuaj.4640

See related article on page $\$ 113$.

$\mathrm{T}$ he role of urodynamics in stress urinary incontinence (SUI) remains controversial. Most published work suggests that preoperative urodynamic studies (UDS) do not improve incontinence surgery outcome in patient with simple stress incontinence. In clinical practice, however, most patients have complex incontinence, sometimes with significant comorbidity. The principle reason for doing the UDS is to make sure the patient needs surgery, to avoid unnecessary complications, to plan for additional management, and to help define the patient's expectations.

Urethral pressure profile (UPP) and abdominal leak point pressure (ALPP) have been used to assess sphincter mechanism of the urethra. Both tests lack proper standardization, which makes it difficult to determine correct cutoff values and consequently, meaningful conclusions. Very low values of both tests may indicate more severe sphincter insufficiency, but again, what are the low cutoff values? Would we change the surgical technique?

The presence of detrusor overactivity (DO) may have some implication on surgery outcome and additional management; however, about $30 \%$ of the patients with mixed stress and urgency incontinence will see improvement in both components after surgery. What about the remaining
$70 \%$ ? It is not clear from the literature how to predict the persistence of DO, or worse, to determine who will develop DO de novo. In general, the presence or absence of DO may not affect outcome significantly, but this knowledge will help in advising the patient about surgery and clarifying expectations. To compound the issue, not all patients with overactive bladder $(\mathrm{OAB})$ will have $\mathrm{DO}$ and not all DO expresses itself as $\mathrm{OAB}$. The issue of underactive detrusor is not well-defined either. There are only a few published papers, offering conflicting conclusions. Is poor detrusor contractility a contraindication for surgery? Should we modify surgical techniques in these patients? What about women with bladder outlet obstruction (BOO)? What is $\mathrm{BOO}$ in women? Should we avoid surgery in these cases? We have more questions than answers, and more research must be done to clarify these issues.

In conclusion, a simple SUI can be managed surgically without UDS; however, in most patients with complex incontinence, the decision regarding UDS has to be made using clinical judgement on a case-by-case basis.

Competing interests: The author reports no competing personal or financial interests.

Correspondence: Dr. Jerzy B. Gajewski, Department of Urology, Dalhousie University, Halifax, NS, Canada; Jerzy.Gajewski@Dal.Ca 